\title{
Thermal Analysis of Zinc Oxide-Eugenol Cements During Setting
}

\author{
H. M. EL-TAHAWI and R. G. CRAIG \\ School of Dentistry, University of Michigan, Ann Arbor, Michigan 48104, USA
}

\begin{abstract}
Characteristic thermal transitions of zinc oxide and eugenol materials were compared with those of zinc eugenolate.
\end{abstract}

It has been accepted traditionally that the setting of zinc oxide-eugenol (ZOE) cements is the result of the formation of zinc eugenolate crystals. ${ }^{1}$ A recent study pointed out that at least seven hours elapsed before zinc eugenolate crystals precipitated from a clear solution of zinc acetate in eugenol. ${ }^{2}$ Accelerated $\mathrm{ZOE}$, however, set in as short a time as three minutes. ${ }^{3}$

Electron microscopy, electron diffraction, ${ }^{2}$ and $\mathrm{X}$-ray diffraction ${ }^{4}$ studies indicated that a set ZOE mix may contain less than $2 \%$ zinc eugenolate. Even that low concentration is doubtful because the studies did not identify clearly whether the diffraction patterns were due to a crystalline or amorphous phase. Differential thermal analysis of set commercial ZOE did not show the characteristic transition of zinc eugenolate on melting. ${ }^{5}$ These studies suggest that the setting of $\mathrm{ZOE}$ cements may not be because of the formation of a zinc eugenolate phase.

Various studies on the same system indicated that the addition of $o$-ethoxybenzoic acid, ${ }^{6}$ large amounts of zinc acetate dihydrate (accelerator), ${ }^{3}$ or zinc eugenolate powder to standard ZOE mixes improved the mechanical properties of the hardened mass.

The purpose of this investigation was to establish the characteristic thermal transitions of $\mathrm{ZOE}$ materials, to compare them to those of zinc eugenolate, and to study the effect of adding $o$-ethoxybenzoic acid, large amounts of accelerator, and rosin on the formation of zinc eugenolate.

This investigation was presented at the 48th General Meeting of the IADR, New York, NY, March 19, 1970.

Received for publication April 13, 1970.

\section{Materials and Methods}

One commercial* cement dispensed as powder and liquid and several laboratory prepared mixes were used in this investigation. The materials used to prepare various cement compositions were as follows: zinc oxide ( $\mathrm{ZnO}), \dagger$ eugenol (E), $\dot{+}$ zinc acetate dihydrate $(\mathrm{ZnAc}), \S$ rosin, $\|$ and o-ethoxybenzoic acid (EBA).\#

Zinc eugenolate was prepared by dissolving zinc acetate in eugenol to form a clear solution. After centrifuging, the clear liquid was left to stand and zinc eugenolate was precipitated from the solution. The precipitate was washed from the eugenol several times with methanol and dried at $45 \mathrm{C}$.

Differential thermal analysis was done at from 25 to $500 \mathrm{C}$ for the various mixes. The analysis was conducted in a nitrogen atmosphere and a heating rate of $25 \mathrm{C} /$ minute was used. Glass beads were used as a reference material in all instances, except for zinc eugenolate in zinc oxide mixtures. Zinc oxide was used as a reference material in these instances. chromelalumel thermocouples were used. The curves showed uncorrected temperatures but those reported in the text are corrected values. The relative humidity of the room during mixing was about $25 \%$.

\section{Results}

Thermograms of zinc oxide, $100 \%$ zinc eugenolate, and $8 \%$ zinc eugenolate in zinc oxide are shown in Figure 1. It can be seen

\footnotetext{
* S.S. White Co., Philadelphia, Pa.

$\dagger$ Analytical reagent, Mallinckrodt, St. Louis, Mo.

U.S.P., Bowler Chem. Co., Detroit, Mich.

$\S$ Reagent grade, J.T. Baker Chem. Co., Phillipsburg,

II Supplied by Kerr Mfg. Co., Romulus, Mich.

\# City Chem. Corp., New York, NY.

DuPont 900 differential thermal analyzer. E.I. DuPont de Nemours \& Co. (Inc.), Instrument Product Division, Wilmington, Del.
} NJ. 


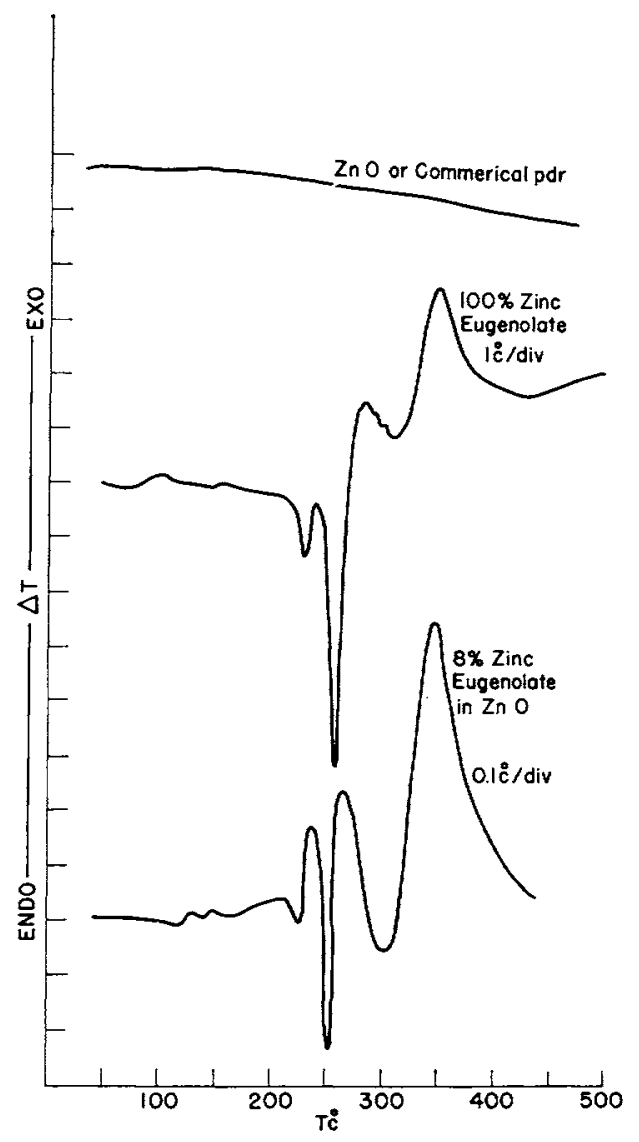

FIG 1.--Zinc oxide, zinc eugenolate, and $8 \%$ zinc eugenolate in zinc oxide.

that within the range of temperature used zinc oxide had no transitions. Zinc eugenolate was characterized by two endothermic and one exothermic transitions; the endotherms were at $226 \mathrm{C}^{*}$ and at $251 \mathrm{C}$, and the exothermic transition was at $348 \mathrm{C}$. The same transitions were detected easily at concentrations of $2 \%$ zinc eugenolate in zinc oxide. Below this concentration only the endotherm characteristic of zinc eugenolate melting ( $251 \mathrm{C})$ was detectable.

The differential temperature $(\Delta \mathrm{T})$ vs the concentration of zinc eugenolate in zinc oxide is shown in Figure 2. The same sample weight $(0.05 \mathrm{gm})$ was used for all the indicated concentrations, and the $251 \mathrm{C}$ endotherm was used to obtain the curve.

Differential thermograms of the reactants

* All temperature values are corrected temperatures.

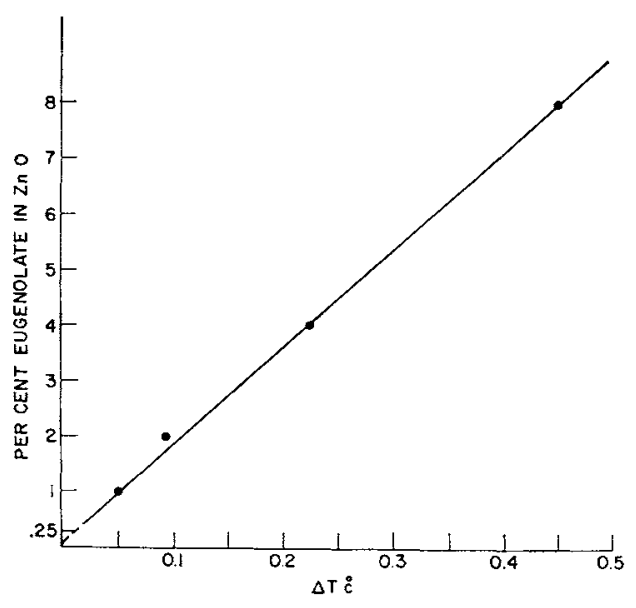

Fig 2.-Differential temperature $(\Delta \mathrm{T})$ vs concentration of zinc eugenolate.

used in preparing the laboratory mixes are shown in Figure 3. Eugenol was characterized by one transition, an endotherm indicating boiling at $253 \mathrm{C}$. Zinc acetate dihydrate showed endotherms at 105, 111,

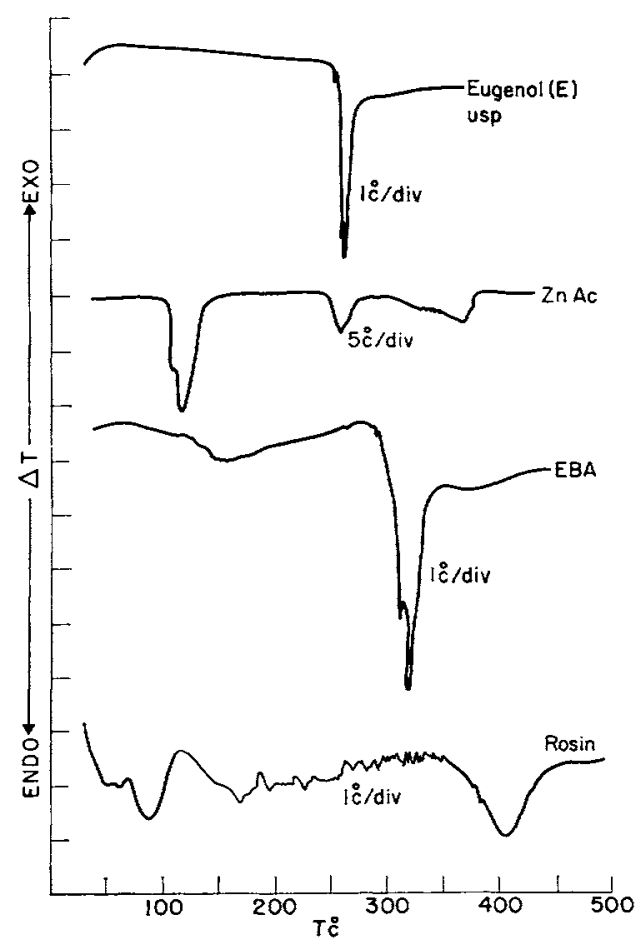

FIG 3.-Components used to prepare ZOE and EBA cements. 
251 , and $355 \mathrm{C}$. The o-ethoxybenzoic acid had an endotherm at $311 \mathrm{C}$. The thermogram of rosin showed endotherms at 84 and $393 \mathrm{C}$.

Differential thermograms of unaccelerated mixes of zinc oxide and eugenol containing no accelerators were obtained at various periods of time from the start of mixing. Up to 96 hours the only transition obtained was that characteristic of the boiling of eugenol, and the mix had not set.

Thermograms of commercial ZOE mixes during and after setting are shown in Figure 4. During setting the mixture showed two endothermic transitions characteristic of the boiling points of water and eugenol. Thermograms of older mixtures indicated a decrease in both endotherms and the simultaneous appearance of two exotherms. A thermogram of a 30-minute-old* mixture showed no endotherms, but exotherms were obtained at 353 and $373 \mathrm{C}$.

Thermograms of laboratory prepared ZOE that contained $0.6 \%$ zinc acetate are shown in Figure 5. It can be seen that this compound had transitions typical of the commercial product used.

The effect of zinc acetate concentration on thermograms of ZOE is shown in Figure 6. As the concentration of zinc acetate was increased an endotherm starts to appear in

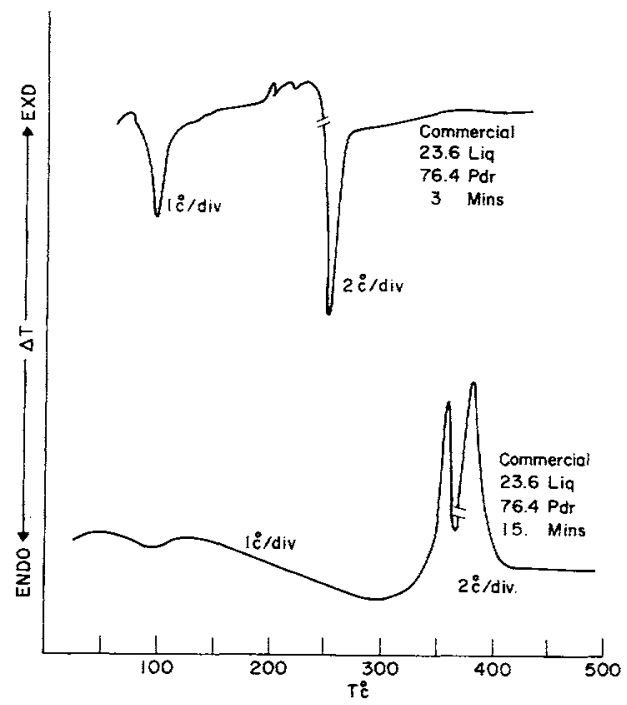

FIG 4.-Commercial ZOE cement.

* Time recorded when run started.

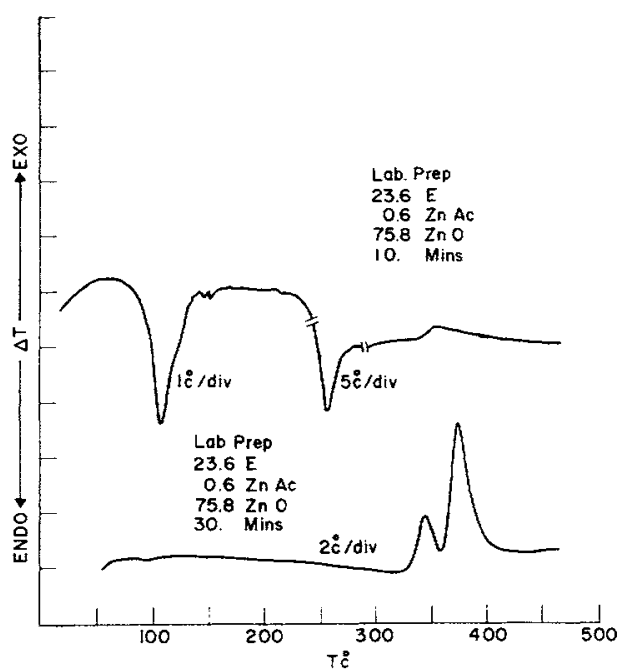

FIG 5.-Laboratory prepared ZOE mixes.

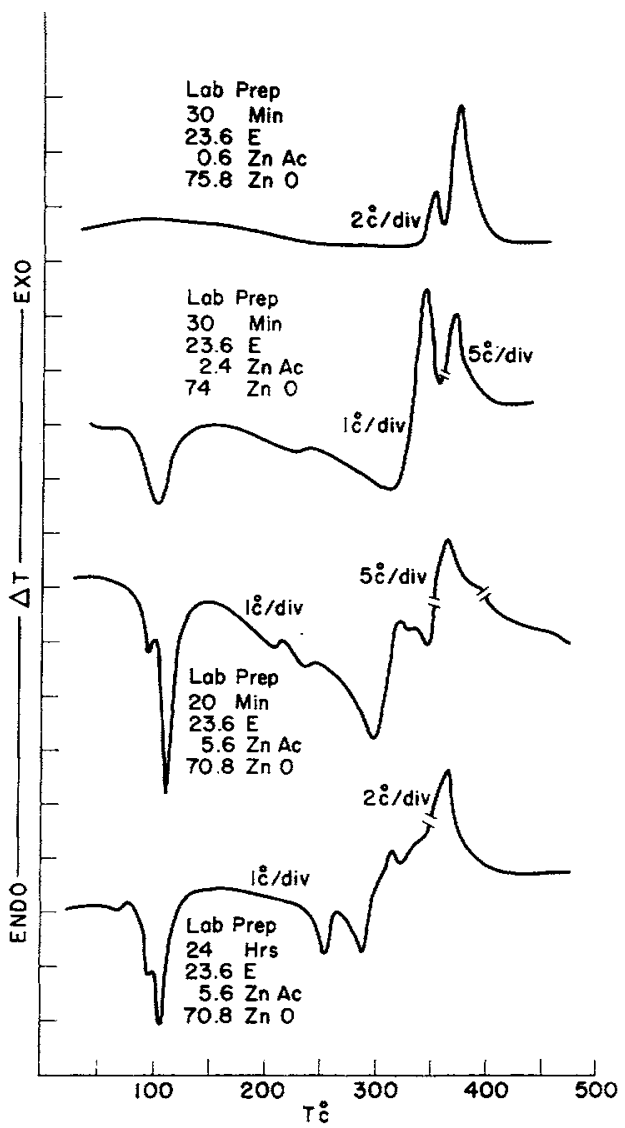

FIg 6.-Effect of zinc acetate concentration on thermograms of ZOE laboratory mixes. 
the region of the melting of zinc eugenolate. The two exotherms at higher temperatures also were replaced by one exotherm. When mix containing a $5.6 \%$ zinc acetate was analyzed after 24 hours, an endotherm appeared at the temperature characteristic of the melting of zinc eugenolate.

The effect of rosin on the reaction between zinc acetate, zinc oxide, and eugenol is shown in Figure 7. The endotherms typical of ZOE mixes with high accelerator concentration were flattened.

Differential thermograms of mixes containing o-ethoxybenzoic acid are shown in Figure 8 . A liquid of $62.5 \%$-ethoxybenzoic acid and $37.5 \%$ eugenol, by volume, had an endotherm at $285 \mathrm{C}$, which was higher than the boiling point of eugenol and lower than that of $o$-ethoxybenzoic acid. Thermograms of the product of the reaction between $o$-ethoxybenzoic acid and zinc oxide had three endotherms at 90,305 , and $425 \mathrm{C}$. In addition to these transitions, a thermogram of the reaction product between zinc oxide, eugenol, and o-ethoxybenzoic acid showed an endotherm at the temperature characteristic of the zinc eugenolate melting point; the lower endotherm found for o-ethoxybenzoic acid and zinc oxide was shifted to $125 \mathrm{C}$.

\section{Discussion}

Thermograms of zinc oxide showed no transitions within the temperature range of interest and therefore could be used as a standard with mixes of zinc oxide and zinc

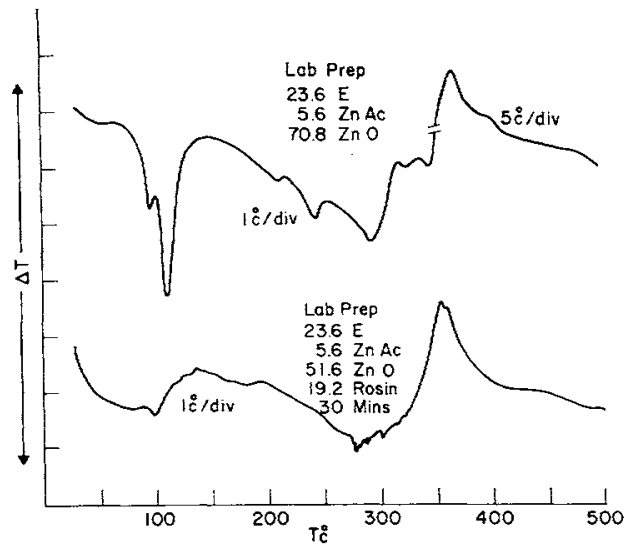

Fig 7.-Effect of rosin on the thermograms of ZOE laboratory mixes.

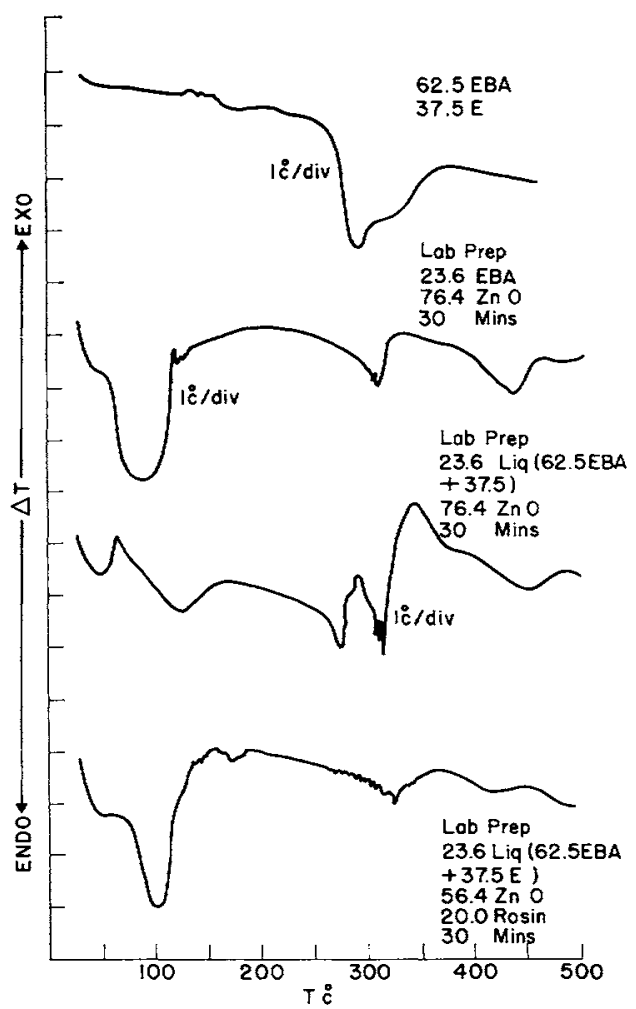

FIG 8.-ZOE cements containing EBA.

eugenolate. The thermograms of zinc eugenolate in Figure 1 had two endotherms and one exotherm; the first endotherm was a solid-solid transition and the second at $251 \mathrm{C}$ was the melting transition. The melting point of zinc eugenolate is reported as 245 C. ${ }^{1}$ The exotherm for zinc eugenolate is interpreted to be a result of decomposition.

The sensitivity of the thermal analysis in detecting zinc eugenolate was estimated to be $0.1 \%$ from Figure 2 and using the assumption that a peak one-eighth inch from the base line could be detected at the highest sensitivity.

Eugenol had one endothermic transition at $253 \mathrm{C}$, which is in agreement with the boiling point of $255 \mathrm{C}$. The endotherms characteristic of zinc acetate dihydrate were due to loss of moisture, loss of water of hydration, melting, and degradation, respectively. The o-ethoxybenzoic acid had one endotherm, which indicated boiling. The two endotherms in the thermograms 
of rosin were because of melting and vaporization.

When differential thermograms of a ZOE formulation containing no accelerator were determined at times of up to 96 hours after the start of mixing, the only transition obtained was that typical of the boiling point of eugenol. This indicates that the reaction between zinc oxide and eugenol alone produced neither zinc eugenolate nor any byproduct that had transitions within the temperature range used.

Thermograms of the commercial cement used during setting had two endotherms due to the loss of water and eugenol. These two endotherms disappeared after 15 minutes from the start of mixing and two exotherms were obtained. The exotherms were at somewhat higher temperatures $(5$ and 25 C) than the temperature for zinc eugenolate decomposition. The thermograms of the set cement were in general agreement with those reported by Brauer, Termini, and Bruns $^{5}$; however, the curves reported had large increases in $\Delta T$ at temperatures just above room temperature. This increase was probably a result of setting the heat programmer at too high an initial value with respect to starting temperature of the sample and is an artifact rather than a real part of the thermogram.

A laboratory cement that contained $0.6 \%$ zinc acetate had transitions typical of the commercial product. Thermograms of both the commercial and laboratory prepared cements were obtained at periods of up to two weeks and the curves were the same. The data presented in Figures 4 and 5 indicate that zinc eugenolate crystals do not form during or after setting of conventional ZOE cements; however, it does indicate the formation of an amorphous phase characterized by an exothermic transition near the decomposition temperature of zinc eugenolate. It should be emphasized that this phase is not characterized by either the solid-solid transition or the melting of crystalline zinc eugenolate.

Increase in the concentration of zinc acetate had two effects on the thermograms of conventional ZOE mixes. The two exotherms of set conventional ZOE were replaced by one exotherm characteristic of the zinc eugenolate, and an endotherm characteristic of the melting of crystalline zinc eugenolate was obtained. The endotherm was more prominent in the thermogram of the mixes 24 hours after setting. These changes suggest the formation of crystalline zinc eugenolate in ZOE mixes that contain high concentrations of zinc acetate, and may explain the finding that the compressive strength of ZOE was increased as a direct function of zinc acetate concentration. ${ }^{2,3}$ The observations that crystalline zinc eugenolate forms as a result of the reaction between zinc acetate and eugenol in a ZOE mix and does not form as a result of the reaction between zinc oxide and eugenol of the same clinical consistency, may be based on the different solubility of zinc acetate dihydrate and zinc oxide in eugenol.

The effect of rosin on the endotherms of ZOE mixes with high concentrations of zinc acetate showed that rosin interfered with the formation of crystalline zinc eugenolate. This may be because of the solution of rosin in eugenol, or the effect of rosin on the precipitation of crystalline zinc eugenolate, or both.

Differential thermograms of o-ethoxybenzoic acid-ZOE cements had endotherms characteristic of the melting of crystalline zinc eugenolate. Thus the addition of $o$-ethoxybenzoic acid to the system encouraged the formation of crystalline zinc eugenolate. The formation of crystalline zinc eugenolate in o-ethoxybenzoic acid-ZOE mixes may be because of the higher solubility of zinc oxide in $o$-ethoxybenzoic acid. The solution of a zinc salt in a liquid brings zinc ions in contact with eugenol to form zinc eugenolate. The observation that crystalline zinc eugenolate improves the compressive strength of $\mathrm{ZOE}^{2}$ cements may explain the efficiency of $o$-ethoxybenzoic acid in improving the strength.

\section{Conclusions}

The experimental evidence furnished by this investigation leads to the following conclusions: (1) The setting of unaccelerated ZOE mixes, or those accelerated with concentrations of less than $1 \%$ zinc acetate, did not result in the formation of more than trace amounts, if any, of crystalline zinc eugenolate. Hence, the setting of these ZOE cements was not due to the formation of such a crystalline phase. (2) Set ZOE mixes had two exotherms in the region of the exotherm for zinc eu- 
genolate, which may indicate the formation of an amorphous zinc eugenolate. (3) The presence of large amounts of zinc acetate in ZOE mixes caused the formation of an appreciable amount of crystalline zinc eugenolate. (4) The addition of o-ethoxybenzoic acid to the system resulted in the formation of crystalline zinc eugenolate and hence improved the mechanical properties. (5) The addition of rosin to o-ethoxybenzoic acid-ZOE cements interfered with the formation of crystalline zinc eugenolate.

\section{References}

1. Copeland, H.I.; Brauer, G.M.; Sweeney, W.T.; and Forziati, A.F.: Setting Reaction of Zinc Oxide and Eugenol, $J$ Res Nat Bur Stand 55:133-38, 1955.

2. El-TahaWI, H.M.: Relationship of Eugenolate Formation to the Setting Mechanism of
Zinc Oxide Eugenol Cements, IADR Dental Materials Group Microfilm, Houston, Texas, March 1969.

3. Mohammed, H.M.: Effect of Some Accelerators on the Setting Time of Zinc Oxide Eugenol Reaction Product, thesis, Northwestern University, April 1967.

4. Norling, B.K., and GreENER, E.H.: X-ray Diffraction Studies of the ZnO-Eugenol Reaction, IADR Dental Materials Group Microfilm, San Francisco, California, March 1968.

5. Brauer, G.M.; Termini, D.J.; and Bruns, C.L.: Characterization of Components of Dental Materials and Components of Tooth Structure by Differential Thermal Analysis, $J$ Dent Res 49:100-110, 1970.

6. BRAuER, G.M.; White, E.E.; and Moshonas, M.G.: The Reaction of Metal Oxides with $O$-ethoxybenzoic Acid and Other Chelating Agents, J Dent Res 37:547-560, 1958. 